\title{
Tobacco control research in managed care: opportunities at the National Cancer Institute
}

\author{
Glen D Morgan
}

A combination of recent scientific advances and social, legal, and public policy developments provides an unprecedented opportunity to reduce the burden of death and disease caused by tobacco use. The National Cancer Institute (NCI) has long played a vital and leading role in the battle against tobacco use. Our research programs have provided critical insights into why people smoke and why they find quitting so difficult. Nevertheless, the historic decline in tobacco use is in jeopardy. Cigar use has increased dramatically. ${ }^{1}$ Smoking in a number of subpopulations, including college students, is more common now than a decade ago. ${ }^{1}$

The NCI recently convened a multidisciplinary group of scientists and experts charged with establishing NCI's tobacco related cancer research priorities for the next five years. The resulting document, the "Tobacco research implementation plan", ${ }^{1}$ outlines nine unique, overarching research opportunities designated as the top priorities. These range from basic biological and biobehavioural research to intervention, epidemiology, and surveillance research.

We believe that tobacco use research can and should be integrated into health care delivery systems. Three of the nine research opportunities appear to have special relevance to managed care settings and industry: treatment, dissemination, and policy. NCI is interested in the development of more powerful treatments and interventions tailored to specific sociocultural, psychological, physiological, and genetic subgroups. Furthermore, intervention programs that capitalise on the dissemination of already proven treatments, perhaps capitalising upon the system features in managed care organisations, would be a significant contribution. Managed care organisations also may be positioned to explore the impact of tobacco policies, such as tobacco use or access restrictions and reimbursement for treatment. The NCI, as well as the Agency for Healthcare Research and Quality (formerly the Agency for
Health Care Policy Research), is very interested in supporting research in these areas within the context of managed care organisations. The infrastructure of managed care organisations allows examination of longitudinal patient data and health outcomes in ways that are very expensive to replicate in "start up" studies. Managed care organisations applying for research funds might be able to enhance their competitiveness for grant funding by forming alliances with academic institutions.

Applications for research grant funding may either be in response to requests for applications (RFAs) or investigator initiated. RFAs designate specific areas of research and outline requirements for applications. The majority of funding from NCI, however, is initiated by investigators. The most common investigator initiated mechanism is the R01, a traditional research project that supports a discrete, specific project. The R03, also known as a small research grant, is a useful mechanism for first time applicants, and has more limited scope and funding than the R01. There are dozens of grant, career award, and training mechanisms available at NCI. "Everything you wanted to know about the NCI grants process" 2 and the website (http://deainfo.nci.nih.gov/) are excellent resources concerning the processes of grant funding. Information about training can be found at: http:/www.nih.gov/training/ extramural.htm Furthermore, program officials in the Tobacco Control Research Branch at NCI (see http://dccps.nci.nih.gov/tcrb/) can assist potential applicants with appropriate mechanisms and provide feedback regarding the applicability of topics.

1 National Cancer Institute. Tobacco research implementation plan: priorities for tobacco research beyond the year 2000. Bethesda, Maryland: Tobacco Research Implementation Group, National Cancer Institute, NIH, November 1998.

2 National Institutes of Health. Everything you wanted to know about the NCI grants process. Bethesda, Maryland: National Cancer Institute, NIH, November 1998. (NIH Publication No (NIH) 98-1222.) 